\title{
Correlated ion analysis and the interpretation of atom probe mass spectra
}

D. W. Saxey

Department of Materials, University of Oxford, Parks Rd, Oxford, OX1 3PH, UK.

Phone: $+44(0) 1865273711$

Fax: $\quad+44(0) 1865273789$

Email: david.saxey@materials.ox.ac.uk

Keywords: atom probe tomography; time-of-flight mass spectrometry; ion dissociation

\begin{abstract}
Several techniques are presented for extracting information from atom probe mass spectra by investigating correlations within multiple-ion detector events. Analyses of this kind can provide insights into the origins of noise, the shape of mass peaks, or unexpected anomalies within the spectrum. Data can often be recovered from within the spectrum noise by considering the time-offlight differences between ions within a multiple event. Correlated ion detection, particularly when associated with shifts in ion energies, may be used to probe the phenomenon of molecular ion dissociation, including the questions of data loss due to ion pile-up or the generation of neutrals in the dissociation process.
\end{abstract}

\section{Introduction}

With the advent of commercial large field-of-view atom probe instruments, numerous studies have been performed to characterize the resolution, accuracy and potential artefacts relating to the spatial reconstruction [1-6]. However, with the mass resolution in such instruments now exceeding 1200 $(1 / \mathrm{FWHM})$, there has been little investigation into similar questions relating to the mass spectra. Although several studies have been performed on compositional accuracy [7-9], other questions such as optimal ranging, noise characterization, and data recovery have received comparatively little attention [10]. Mass spectrum interpretation is critical to several aspects of atom probe tomographic analysis, such as optimization of the acquisition conditions and accurate compositional measurement. This work demonstrates that correlations between ions that are generated and detected on a single pulse can be used to help characterize the mass spectra and gain an improved understanding of highfield processes relevant to atom probe tomography.

The composition of multiple-ion detection events during an atom probe experiment does not usually correspond with the overall sample composition, mainly because some species are preferentially evaporated within multiple-ion events [11]. Furthermore, ionic species composing the detected multievent population are not uniformly distributed among the evaporation events but tend to cluster in 
preferred combinations, an effect often referred to as sympathetic evaporation $[12,13]$. For example, in steels, interstitial elements such as phosphorus, boron and carbon are evaporated mostly in multiple events [14], with boron very likely to be evaporated with other boron or carbon ions. Likewise, oxides, nitrides, carbides, and compound semiconductors show a strong preference for multi-ion field evaporation among particular species $[11,15,16]$. This species-dependent tendency toward detection within multiple-ion events is usually observed within a mass spectrum containing molecular or cluster ions consisting of the same species [17].

Such a bias toward detection within multiple-ion events may introduce compositional inaccuracies into the analysis due to detector dead-time effects, also referred to as ion pile-up or detector saturation [18]. Within a multiple-ion evaporation event, ions arriving at the detector closely-spaced in time may not be distinguished due to a finite relaxation time or confusion of signals at the detector $[19,20]$. This effect is compounded by the fact that multiple-ion evaporation events are highly spatially localized [21] and so impact the position-sensitive detector in a relatively small region. The experimental conditions during an atom probe acquisition are therefore usually optimised so as to minimise multiple-ion detector events.

Despite these drawbacks, the non-random character of multiple-ion events raises the possibility that useful information may be gleaned from them. Recent work has shown that improvements in the signal-to-noise ratio can often be gained by limiting the analysis to include only multiple-ion events within a data set $[13,14]$. The present work makes use of correlations between ions detected on a single pulse event to provide new insights into the nature of mass spectrum noise, the occurrence of molecular ion dissociation, and questions of compositional accuracy. The results presented here were obtained using LEAP 3000X HR ${ }^{\mathrm{TM}}$ and LEAP $3000 \mathrm{X} \mathrm{Si}^{\mathrm{TM}}$ atom probe microscopes, operating in both high-voltage and laser pulsing modes. The laser illumination has a wavelength of $532 \mathrm{~nm}$, with a spot diameter below $10 \mu \mathrm{m}$, and a pulse duration of about 10ps. The detector efficiency is approximately $55 \%$ for the $3000 \mathrm{X} \mathrm{Si}$ instrument, and $37 \%$ for the $3000 \mathrm{X}$ HR. Reconstruction of the atom probe data was performed using the IVAS ${ }^{\mathrm{TM}}$ software package. IGOR Pro 6.1 was used for data analysis and presentation.

\section{Analysis techniques}

\subsection{Correlation tables}

The correlation between ion pairs occurring within the same multi-hit event can be measured by constructing a 'correlation table'. The approach used here is to compare the frequency of ion-pair coincidences within multiple-ion events with an expected distribution based on a hypothesis of independent evaporation. First, the population of ion-pairs, $p_{i j}$, is generated by considering every pair-combination within each multiple-ion event. An n-multiple event contains $n(n-1) / 2$ pairs, and 
the ion order is not considered so that $p_{i j}=p_{j i}$. Using a contingency table-type approach, a corresponding matrix of expectation values $\left(e_{i j}\right)$ is generated, under the assumption that pairs are formed by independent evaporation of the constituent ions. As for a contingency table analysis, the row and column totals can be used to calculate the probability that a given ion-pair will contain an ion of type $k$.

$$
P(k)=\frac{\sum_{j} p_{k j}}{\sum_{i j} p_{i j}}=\frac{\sum_{i} p_{i k}}{\sum_{i j} p_{i j}}=\frac{n_{k}}{N},
$$

where $n_{k}$ is the sum of the $k^{\text {th }}$ row or column and $N$ is the total number of pairs in the table. The expectation values are then given by

$$
e_{i j}=P(i, j) N=P(i) P(j) N=\frac{n_{i} n_{j}}{N} .
$$

Other means, such as a priori techniques, can also be used to calculate $e_{i j}[22,23]$. The significance of any deviation between the observed and expected pair counts is then quantified by

$$
d_{i j}=\frac{p_{i j}-e_{i j}}{\sqrt{e_{i j}}}
$$

where the denominator represents an estimate of the standard deviation of the observed values from the expectation value, assuming Poissonian statistics. The $d_{i j}$ values are therefore a measure of the extent to which the observations depart from the expected values under the hypothesis of independent evaporation.

It is important to recognise that these values $\left(d_{i j}\right)$ express the relative correlations and anticorrelations between the various ion species. Therefore if ion type $m$ is strongly correlated with ion type $n$, the other ions in row $m$ or column $n$ will tend to show anti-correlations, and vice versa. This leads to a bias in the analysis of real atom probe data as the self-ion correlations $\left(p_{i i}\right)$ are inevitably under-represented due to ion pile-up effects at the detector. As a result, off-diagonal ion-pairs tend to show positive correlations that are misleading. To avoid this effect, the actual observed diagonal $\left(p_{i i}\right)$ values can be ignored and instead replaced by values that are artificially generated to be equal, or nearly equal, to their corresponding expected values: $p_{i i} \cong e_{i i}$. This requires an iterative adjustment of the $p_{i i}$ values, based on the off-diagonal values in the remainder of the table. The $p_{i i}$ converge quickly toward the $e_{i i}$ values, and the effect is to remove the influence of the diagonal counts on the remaining, off-diagonal, ion-pair correlations. This procedure eliminates any potential information gained from the self correlations, but ensures that the diagonal terms are neutral in the analysis and do not bias the other $d_{i j}$ values. The $i^{\text {th }}$ row or column should therefore be interpreted as showing the relative amounts of correlation between ion-type $i$ and every other ion-type except $i$ itself. 
An example of such an analysis on pure aluminium is shown in Figure 1. Even for this simple system, correlations in field evaporation are evident. The association of $\mathrm{H}$ with $\mathrm{H}_{2} \mathrm{O}$ species is probably the result of contamination of the specimen surface by water vapour. The most striking correlation is seen in the $\mathrm{AlH}^{+}$ions, which are very heavily weighted toward evaporation with $\mathrm{H}^{+}$- perhaps resulting from a dissociation of $\mathrm{AlH}_{2}{ }^{2+}$, which is also present in the spectrum in the singly-charged state. Based on the number ion-pairs, $n_{i}$, associated with each ion-type, $i$, the expected number of $\left\{\mathrm{AlH}^{+}, \mathrm{H}^{+}\right\}$pairs was 71 (Eq. (2.2)). The observed number was 827. Conversely, the expected number of $\left\{\mathrm{AlH}_{2}{ }^{+}, \mathrm{H}^{+}\right\}$ pairs was 10, but none were observed.

This treatment may be extended further by considering additional ions and looking at correlations between triplets or higher-order groupings. In practice full multi-dimensional analyses require very large data sets and are difficult to visualize. However analysis of higher-order correlations can be performed easily for specific ion combinations. For example, by first selecting a highly-correlated ion-pair from within a correlation table and looking for correlations with other ions within this subpopulation of multiple events the most highly-correlated species can be determined. This was performed in the case of a Fe-Cr oxide analysis, in which $\mathrm{Fe}^{+}$ions were found to be strongly associated with $\mathrm{Fe}^{2+}$ ions. Using the relative abundances of each species within the total multiple-ion event population, an expectation value can be generated for the number of occurrences of each species within the sub-population of $\mathrm{Fe}^{+}+\mathrm{Fe}^{2+}$ multiples. Statistical deviation values can then be calculated, as for the correlation table, using an analogue to Eq. (2.3). In this case the multiple events containing $\mathrm{Fe}^{+}$and $\mathrm{Fe}^{2+}$ were found to be strongly enriched in $\mathrm{O}^{+} / \mathrm{O}_{2}{ }^{2+}$ and $\mathrm{O}_{2}{ }^{+}$(Figure 2), possibly resulting from dissociation of $\mathrm{Fe}_{2} \mathrm{O}_{(2)}$.

By themselves the ion-pair correlations do not endorse any particular underlying mechanism leading to their appearance. Assuming they are not explained by instrumental causes, the correlations may be accounted for by sympathetic co-evaporation of different species from the surface on the same pulse, or by the field evaporation and subsequent dissociation of a molecular ion. However, if this analysis is extended to smaller, contiguous mass ranges, so that a two-dimensional spectrum, or 'correlation histogram', is created, then information may be gained on the underlying mechanisms leading to the ion-pair correlations.

\subsection{Correlation histograms}

Rather than limiting the analysis to particular mass ranges, pair correlations within multi-ion events can be visualized across the entire mass spectrum by plotting each ion-pair in a two-dimensional histogram. Such a correlation histogram is shown in Figure 3 for the evaporation of (0001) gallium nitride under low-power laser pulsing conditions $(0.03 \mathrm{~nJ} /$ pulse); at an average evaporation rate of $3 \mathrm{x}$ $10^{-2}$ detected ions per pulse and a base temperature of $26 \mathrm{~K}$. As for the correlation tables, the ion-pair 
order is not considered and the displayed histogram is diagonally symmetric. In this case it is the $p_{i j}$, pair-abundance values themselves that are shown, rather than the deviation values, $d_{i j}$. The latter may also be generated to provide complimentary information on the significance of features within the histogram. The number of pairs in each 2-dimensional histogram bin is indicated by its colour.

Unsurprisingly, the majority of the histogram counts are located at coincidences between major peaks $(i$ and $j$ ) in the mass spectrum. However, the most obvious features of the histogram are the lines that emanate from these coincident points. The horizontal and vertical lines represent the field evaporation of ion-type $i$, coincident with the pulse, followed by evaporation of the paired ion, $j$, at some time, $\Delta t$, after the pulse. The diagonal trails, moving from lower-left to upper-right, reflect the simultaneous evaporation of ions $i$ and $j$ from the specimen surface at some time, $\Delta t$, after the pulse. Their times-offlight are therefore each extended by $\Delta t$, and they fall along a slightly curved track in the correlation histogram. It is clear from comparison with the conventional mass spectrum that these tracks, whose intensity falls-off with distance from the main coincidence point $(\Delta t=0)$, correspond to the tails following each mass peak. In the conventional mass spectrum these peak tails overlap with each other, making it difficult to perform a deconvolution. However, the pair-wise correlation histogram separates these tails in two dimensions and provides a clearer picture of the processes underlying the spectrum noise.

Three correlation histograms generated from the field evaporation of a bulk metallic glass sample under different conditions are presented in Figure 4. They illustrate the understanding that may be gained from this approach. The data in Figure 4(a) was obtained in voltage pulsing mode, at a pulse fraction of 0.20 , an ion detection rate of $5 \times 10^{-3} /$ pulse, and a temperature of $50 \mathrm{~K}$. Although only weak diagonal trails are observed following each peak in the histogram, they extend throughout the entire pulse repetition time-period, in this case $5 \mu \mathrm{s}$, and into the following pulse window. Many correlated field evaporation events occur at times well away from the voltage pulse, and these are responsible for the significant background noise observed in the mass spectrum. Detector noise and the evaporation of contaminant species do not contribute significantly to this background. This suggests that the effective pulse fraction may be much lower than 0.20 , possibly due to poor electrical conductivity in the sample mount. It also raises the possibility of determining the chemical composition of the background noise counts, at least for multi-ion events, by indentifying ion species from their corresponding counts in the correlation histogram tails. Figure 4(b) shows data from the same material under laser pulsing. The average energy per pulse is $0.1 \mathrm{~nJ}$, the ion detection rate is $10^{-2} / \mathrm{pulse}$, and the base temperature is $50 \mathrm{~K}$. In this case the tails are more strongly defined and more confined to the histogram peaks, indicating that evaporation is tied more closely to the pulse. There is a significant 'cloud' of hits in the histogram to the upper-right of each major coincident point, which corresponds to ions evaporated after the pulse but not in concert with other ions that may leave the surface 
following the same pulse. This indicates that field-evaporation events within the mass spectrum tails are not well correlated, which is consistent with the low-field conditions expected from excess heating of the specimen by the laser pulse. The histogram in Figure 4(c) was obtained from field evaporation of the specimen without any pulsing, often termed "dc evaporation". The departure of ions from the surface is still highly correlated in time, giving rise to the tracks that are clearly visible in the histogram and suggesting that ion identities may be extracted, even in the absence of pulsing.

\subsection{Signatures of molecular ion dissociation}

Studies of correlations within multiple-ion events can also be used to probe ion dissociation processes that may occur after field evaporation. If a molecular ion is field evaporated in a metastable state and subsequently decomposes into two or more particles [24-26] the products will have slightly different flight times compared with ions of equivalent mass and charge evaporated directly from the surface. If the molecule dissociates more than a few nanometres from the surface, then the resulting time-offlight differences may be sufficiently large to be observed in a high-resolution mass spectrum. Furthermore, a particular relationship will exist between the energy shifts experienced by each fragment ion due to their common history within the parent ion.

An ion of mass $M_{1}$ and a charge $q_{1}$ will gain a kinetic energy $E_{1}$ following field evaporation from a specimen at a potential $V_{0}$ :

$$
E_{1}=\frac{1}{2} M_{1} v_{1}^{2}=q_{1} V_{0}
$$

This fixes the final (zero potential) velocity, $v_{1}$, in terms of the mass-charge ratio $m_{1}=M_{1} / q_{1}$ and the potential,

$$
v_{1}^{2}=\frac{2 V_{0}}{m_{1}}
$$

Under the assumption of rapid ion acceleration followed by a long field-free drift toward a grounded detector, this velocity, and therefore the mass-charge ratio, can be determined from the time-of-flight over a fixed path length. This is the basis for simple time-of-flight mass spectrometry.

As illustrated in Figure 5, suppose that a molecular ion is field evaporated from the surface of a specimen at a potential $V_{0}$, with a mass-charge ratio $m_{p}$. After travelling away from the surface through a potential drop of $V_{d}$, the ion dissociates, yielding at least two daughter ions having masscharge ratios of $m_{1}$ and $m_{2}$. Due to the initial period of flight as a constituent of the parent ion, the final velocity of the first daughter ion will be modified from $v_{1}$ to $v_{1}^{\prime}$, yielding a mass-charge ratio measurement of $m_{1}^{\prime}$. This shift, $m_{1}^{\prime}-m_{1}$, can be calculated by equating the potential drop following dissociation with the kinetic energy gain: 


$$
q_{1}\left(V_{0}-V_{d}\right)=\frac{1}{2} M_{1} v_{1}^{2}-\frac{1}{2} M_{1} v_{d}^{2}
$$

where $v_{d}$ is the velocity of the parent ion at the moment of dissociation. From Equation (2.5):

$$
v_{1}^{\prime 2}=\frac{2 V_{0}}{m_{1}^{\prime}} \text {, and } v_{d}^{2}=\frac{2 V_{d}}{m_{p}}
$$

Combining Equations (2.6) and (2.7):

$$
\begin{aligned}
& \frac{q_{1}}{M_{1}}\left(V_{0}-V_{d}\right)=\frac{V_{0}}{m_{1}^{\prime}}-\frac{V_{d}}{m_{p}}, \\
& \frac{V_{0}}{m_{1}^{\prime}}=\frac{\left(V_{0}-V_{d}\right)}{m_{1}}+\frac{V_{d}}{m_{p}}=\frac{1}{m_{1}}\left[V_{0}-V_{d}\left(1-\frac{m_{1}}{m_{p}}\right)\right] \\
& m_{1}^{\prime}=m_{1}\left[1-\frac{V_{d}}{V_{0}}\left(1-\frac{m_{1}}{m_{p}}\right)\right]^{-1} .
\end{aligned}
$$

For a particular dissociation pathway, $m_{p} \rightarrow m_{1}+m_{2}$, the observed mass-charge ratios of the daughter ions, $m_{1}^{\prime}$ and $m_{2}^{\prime}$, are therefore determined by a single variable, the fractional potential drop $V_{d} / V_{0}$. These daughter ion-pair correlations will therefore form a line, or track, in the correlation histogram that is parameterised by $V_{d} / V_{0}$. Such 'dissociation tracks' begin at the $\left(m_{1}, m_{2}\right)$ ion-pair coincident point, corresponding to $V_{d}=0$, and follow a curved path to the point $\left(m_{p}, m_{p}\right)$, where $V_{d}=V_{0}$. The beginning of the track is equivalent to direct evaporation of the daughter ions from the specimen surface $\left(m_{1}^{\prime}=m_{1}\right.$ and $\left.m_{2}^{\prime}=m_{2}\right)$, and the end-point is equivalent to the absence of dissociation $\left(m_{1}^{\prime}=m_{p}\right.$ and $\left.m_{2}^{\prime}=m_{p}\right)$.

Such tracks within correlation histograms, signifying molecular ion dissociation, have been observed in data obtained from several material systems, including borides, carbides and $\mathrm{Fe}-\mathrm{Cr}$ oxides. Figure 6 shows part of a correlation histogram generated from field evaporation of (0001) gallium nitride. Clearly visible are the horizontal and vertical trails connected with the main ion-pair coincident points, and the diagonal trails, corresponding to delayed evaporation, moving from the lower-left toward the upper-right. In addition to these there are also several tracks leading from the upper-left toward the lower-right, as might be expected for histogram counts arising from dissociation events. Also plotted on the histogram are three sets of parametric curves, based on Equation (2.8), corresponding to the dissociation tracks expected for the fragment ions of three different molecular ion dissociation pathways:

(1) $\mathrm{GaN}^{2+} \rightarrow \mathrm{Ga}^{+}+\mathrm{N}^{+}$

(2) $\mathrm{GaN}^{3+} \rightarrow \mathrm{Ga}^{2+}+\mathrm{N}^{+}$

(3) $\mathrm{GaN}_{3}^{2+} \rightarrow \mathrm{Ga}^{+}+\mathrm{N}_{3}^{+}$. 
Given the simplicity of the model, and the fact that a limited selection of mass-charge ratios are available for $m_{1}, m_{2}$ and $m_{p}$, the agreement with the observed tracks in the correlation histogram is remarkably close. Furthermore these particular dissociation pathways appear physically reasonable. In each case mass and charge are conserved in the dissociation, though this conservation is not a constraint of the model used to derive Equation (2.8). The dissociations all involve breaking of Ga-N bonds, which are expected to be weaker than the N-N bonds in the $\mathrm{GaN}_{3}$ molecular species [27].

Another striking feature of the dissociation tracks is their variable intensity, both along their length and between tracks belonging to different dissociation pathways. Dissociation appears to be occurring at preferred locations and times following the initial field evaporation event. For example dissociation of $\mathrm{GaN}^{2+}$ mainly occurs close to the specimen surface, while $\mathrm{GaN}_{3}{ }^{2+}$ does not appear to dissociate until reaching regions of much lower potential. As shown by Tsong et al. [24,28], this behaviour may be expected due to the highly complex dissociation dynamics possible following field evaporation of molecular ions from an atom probe specimen.

For the dissociation tracks (1) and (3) in Figure 6, the parent ions are clearly present in the mass spectrum, indicating either incomplete dissociation of identical molecular ions, or the field evaporation of some parent ions in a metastable high-energy state that decays by dissociation after a short time. The short dissociation track (2) terminates at the parent ion, $\mathrm{GaN}^{3+}$, which is not observed in the mass spectrum or in the correlation histogram.

If the dissociation of a particular molecular ion always occurs soon after field-evaporation, then only a short dissociation track may be visible radiating from the ion-pair coincident point in a correlation histogram. The parent-ion identity may still be inferred from the angular orientation of this track using Equation (2.11), below. If the dissociation occurs close to the specimen surface, then $V_{d}<<V_{0}$, and a binomial approximation can be made in Equation (2.8), yielding

$$
m_{1}^{\prime} \cong m_{1}\left[1+\frac{V_{d}}{V_{0}}\left(1-\frac{m_{1}}{m_{p}}\right)\right]
$$

The shift in the measured mass-charge ratio is then

$$
\Delta m_{1}=m_{1}^{\prime}-m_{1}=m_{1} \frac{V_{d}}{V_{0}}\left(1-\frac{m_{1}}{m_{p}}\right)
$$

Therefore, assuming early dissociation, the measured mass-shift of each daughter ion is proportional to the fractional potential drop $V_{d} / V_{0}$. In this case the curved dissociation track, parameterized by $V_{d} / V_{0}$, approximates to a straight line emanating from the ion-pair coincident point and having a particular angle $\theta$ with respect to the $m_{l}$ axis (' $\mathrm{x}$ '-axis in figures): 


$$
\tan \theta=\frac{\Delta m_{2}}{\Delta m_{1}}=\frac{m_{2}}{m_{1}}\left(\frac{m_{p}-m_{2}}{m_{p}-m_{1}}\right)
$$

The arguments leading to Equations (2.8) and (2.11) assume that no significant kinetic energy is imparted to the daughter ions in the dissociation process. However, there are no assumptions regarding the total number of dissociation products or their charge states - except that any ionization of the daughter ions must occur simultaneously with the dissociation. If it is now assumed that only two products are formed by the dissociation and that it occurs sufficiently far from the surface to preclude post-ionization [29], then ion mass $\left(M_{p}=M_{1}+M_{2}\right)$ and charge $\left(q_{p}=q_{1}+q_{2}\right)$ are conserved, and Equation (2.11) simplifies to

$$
\tan \theta=-\frac{m_{2}}{m_{1}} \frac{q_{1}}{q_{2}}
$$

which is always negative. If $m_{1} \neq m_{2}$, and $m_{2}$ is chosen to be the larger of the daughter ion masscharge ratios, then $m_{1}<m_{p}<m_{2}$ and the dissociation track moves to the lower right in the correlation histogram, in relation to $\left(m_{1}, m_{2}\right)$.

This can be easily understood intuitively. The daughter ion with the lower mass-charge ratio is effectively slowed down during its initial trajectory as a constituent of the parent ion, since $m_{p}>m_{1}$. After dissociation it therefore arrives at the detector later than expected and registers a higher masscharge ratio, $m_{1}^{\prime}>m_{1}$. Conversely the daughter ion having the higher mass-charge ratio moves more quickly than it otherwise would, up until the dissociation of the parent ion. It therefore arrives at the detector earlier than expected and registers a lower mass-charge ratio, $m_{2}^{\prime}<m_{2}$. In a correlation histogram in which $m_{2}^{\prime}$ is plotted against $m_{1}^{\prime}$, ion-pairs corresponding to daughter ions from this type of dissociation event will therefore fall to the lower right of the ion-pair coincident point $\left(m_{1}, m_{2}\right)$.

\section{Discussion}

\subsection{Estimating compositional bias - correlation tables}

The analysis techniques presented here can be used to estimate the quantity, and composition, of data that may be missed in an atom probe acquisition due to detector dead time effects. If such effects are not present, generating a correlation table for each isotope of a particular ion species should result in only small deviation values since the hypothesis of independent co-evaporation would be expected to hold for different isotopes of the same ion species. However, due to ion pile-up at the detector, the self-isotope correlations, $p_{i i}$, will tend to be underrepresented. The number of ions that are missing may be estimated by comparing the observed number of self-isotope pair correlations, $p_{i i}$, with the expected number, $e_{i i}$. As discussed in Section 2.1, only the off-diagonal (mixed-isotope) pair correlations should be used to generate the $e_{i i}$ values, or else they will be affected by the incorrect self-isotope abundances. Some dead-time effects may also exist between different isotopes, though 
this should also be quantifiable from the correlation table if a sufficient number of mixed isotope pairs are unaffected.

\subsection{Estimating compositional bias - correlation histograms}

Although the above method may result in a good estimate for the missing proportion of a particular species, at least within the subset of data obtained from multiple-ion events, it can only be performed on a relatively large number of ions. Therefore any small-scale spatial variations within the reconstruction will be averaged out.

In principle, careful analysis of the correlation histogram can provide information that would be otherwise hidden within the spectrum noise, and even allow partial recovery of data at the level of individual ions. For example, in Figure 3(b), the diagonal trails in the correlation histogram, corresponding to delayed co-evaporation of species following the laser pulse, are convoluted in the mass spectrum and form an overlapping combination of mass peak tails with mixed compositions. In the correlation histogram, the trails are separated-out in a manner that allows individual ion events to be confidently identified. For example, every histogram count within the diagonal trail beginning at the $\mathrm{N}_{2}{ }^{+}-{ }^{69} \mathrm{Ga}^{+}$coincidence point in Figure 3(b) represents two detector events, within the same pulse period, that can be identified as $\mathrm{N}_{2}^{+}$and ${ }^{69} \mathrm{Ga}^{+}$. However in the mass spectrum they are likely to be considered as noise, or incorrectly identified as belonging to other mass peaks.

In the mass-charge correlation histograms presented here, these diagonal trails containing ion-pair correlations of known identity are difficult to isolate due to their curvature. This curvature arises because the histogram is constructed from the mass-charge ratios $(m)$, rather than the times-of-flight, of the ions. Correlated ion-pairs within these trails contain a common addition $(\Delta t)$ to the time-offlight of each ion, but different additions to $m$, since $t \propto \sqrt{m}$. Plotting $t_{2}$ versus $t_{2}-t_{1}$ yields a correlation histogram with straight, vertically aligned trails that can be easily selected for analysis by ranging in $t_{2}-t_{1}$. In this manner, the time-of-flight differences between ion-pairs arriving within the same pulse window can be exploited to identify each ion in the pair. In principle, evaporation events may be recovered, even if they are not coincident with the voltage/laser pulse, and the identified ions can be added to the data to determine how the overall composition is affected.

While the numbers of counts may be small compared to the main peaks in the mass spectrum, and only ions from multiple-detection events can be used, this approach may provide valuable information on chemical biases in cases of preferential evaporation, or where the mass peak tails are particularly large and a high proportion of the ions are collected within multiple-ion events. When the quantification of a multi-isotope species is limited by the detector deadtime, minor isotopes may be used to correct the data [30]. Further improvements in mass resolution and detector efficiency would allow more to be gained from this type of correlation analysis. 


\subsection{Molecular ion dissociation}

The histogram counts represented in the dissociation signatures presented here are typically unranged, or mis-ranged in the data analysis. However, due to their low numbers, they do not significantly alter the measured composition. For example, the dissociation track sections shown for $\mathrm{GaN}^{2+}$ in Figure 6 (inset (1)), contain about 6,000 counts. Since counts in the correlation histograms require the detection of two ions, the actual occurrence of ion dissociation in this region is estimated to be $6,000 \times\left(\frac{1}{\epsilon}\right)^{2} \cong$ 20,000 , where the detector efficiency, $\epsilon$, is assumed to be $55 \%$. The number of ions field evaporated in this data set is approximately 34 million, so the rate of occurrence for this type of dissociation is about 600 per million evaporated ions. How this number changes with the acquisition conditions is not known.

The dissociation tracks in the correlation histograms may provide insights into other possible mechanisms of data loss. For example, many of the tracks reveal that dissociation events are occurring after the potential has fallen by more than $95 \%$, where the field is therefore very low, suggesting that the parent molecular ions are stabilized by the field.. In these late dissociation events, the apparent mass-charge ratios of the daughter ions ( $m_{1}^{\prime}$ and $\left.m_{2}^{\prime}\right)$ are not sufficiently separated to be reliably detected, due to the detector dead-time, and it is not possible to quantify the resulting data loss. Counteracting this potential loss is the fact that ion fragments from such late dissociations have a similar mass-charge ratio to the parent ion. So, if exactly one of the fragments is detected, the measured composition will be unchanged. Whether late dissociations give rise to a deficiency or excess in the parent ion abundance will depend on the particular detector and flight path arrangement.

Another relevant mechanism of data loss may arise from the generation of neutral atomic species during molecular ion dissociation within the ion flight path [31]. While neutral species may reach the detector in a straight-flight-path instrument, if the dissociation occurs within a significant electric field, the measured time-of-flight will be shifted far from the expected mass peak. If the dissociation occurs close to the specimen surface then the neutral atom may not have sufficient energy to trigger the detector.

Evidence has been found for the generation of neutrals arising from dissociation of molecular ions during the field evaporation of gallium nitride. Although not highlighted in Figure 6, a track exists in the correlation histogram corresponding to the dissociation pathway

$$
\mathrm{GaN}_{3}^{2+} \rightarrow \mathrm{Ga}^{+}+\mathrm{N}^{+}+\mathrm{N}_{2}
$$

where the simultaneous generation of a neutral $\mathrm{N}_{2}$ molecule is inferred from the composition of the parent ion and by assuming conservation of charge. No evidence of a charged (post-ionized) $\mathrm{N}_{2}$ species was found in association with these events. However, it appears unlikely that this represents a 
substantial mechanism for nitrogen loss in the field-evaporation of gallium nitride as it only accounts for 100-1000ppm of the nitrogen in the data sets presented here.

No dissociation tracks in any material have revealed post-ionization of the fragment ions, presumably because the dissociation, if visible in the correlation histogram, occurs too far from the specimen surface for this mechanism to be active.

Evidence of dissociation is not observed in data acquired using the LEAP $3000 \mathrm{X}$ HR, due to the energy compensating action of the wide-angle reflectron. The times-of-flight of particles having lower/higher energies than would be expected for their mass-charge ratio experience shortened/extended flight paths through the reflectron, thereby compensating for the energy discrepancy. The flight paths of daughter ions emerging from a dissociation event are therefore modified so as to yield a measured time-of-flight close to that expected for ions of the same masscharge ratio originating from the specimen surface. In some cases a very small 'lobe' $\left(\mathrm{V}_{\mathrm{d}}<0.02 \mathrm{~V}_{0}\right)$ was visible in place of the dissociation tracks in data acquired from the LEAP $3000 \mathrm{X}$ HR, but the effect on the mass spectrum peaks was insignificant.

\section{Conclusions}

The analysis methods described here provide tools for extracting information from the significant correlations that exist between field-evaporated ions in an atom probe experiment. The quantitativeness of these methods is affected by their limitation to multiple-ion events, and only preliminary results and speculation on possible uses are presented here. However, they provide insight into the processes underlying noise and data loss in the mass spectra and show promise for further application to a variety of current problems.

Statistical analysis of ion-pairs within multiple-hit detector events can yield significant information relating to compositional biases. Correlations between ion-types can be difficult to interpret by themselves, because of the number of potential mechanisms in play. However a spectrum-wide approach using 'correlation histograms' can provide more detailed information, and even give access to the identities of ions that are not field-evaporated with the laser or voltage pulse.

The occurrence of molecular ion dissociation can also be determined from the ion correlations. The parent and daughter ions can very often be identified with a high degree of confidence, allowing the presence of other dissociation products to be inferred, including the generation of neutral species. In this way, the correlation histogram opens a window on the dissociation dynamics of molecular ions within atom probe analyses, and may be a useful tool for understanding the mechanisms behind preferential loss of ion species, as known to exist in many systems [15,16,32-34]. 


\section{Acknowledgements:}

The author is grateful to several colleagues for useful discussions surrounding this work, and for comments on the manuscript: Baptiste Gault, George Smith, Daniel Haley, Emmanuelle Marquis, Alfred Cerezo, and Hans-Jürgen Kreuzer. The analysis included data from materials supplied by Dr Kevin Laws of the University of New South Wales, Prof. Colin Humphreys of Cambridge University, and the Institute for Nuclear Safety Systems, Japan. The Fe-Cr oxide specimen was prepared by Karen Kruska. Atom probe analyses were performed at the UK Atom-probe Facility at the University of Oxford - funded by the UK Engineering and Physical Sciences Research Council (EPSRC) under grant no. EP/077664/1. 


\section{References}

[1] B. Gault, M.P. Moody, F. De Geuser, A. La Fontaine, L.T. Stephenson, D. Haley, et al. Spatial resolution in atom probe tomography, Microscopy and Microanalysis. 16 (2010) 99-110.

[2] M.P. Moody, B. Gault, L.T. Stephenson, D. Haley, S.P. Ringer. Qualification of the tomographic reconstruction in atom probe by advanced spatial distribution map techniques, Ultramicroscopy. 109 (2009) 815-824.

[3] B. Gault, M.P. Moody, F. de Geuser, G. Tsafnat, A. La Fontaine, L.T. Stephenson, et al. Advances in the calibration of atom probe tomographic reconstruction, J.Appl.Phys. 105 (2009) 034913.

[4] E.A. Marquis, F. Vurpillot. Chromatic aberrations in the field evaporation behavior of small precipitates, Microscopy and Microanalysis. 14 (2008) 561-570.

[5] B.P. Geiser, T.F. Kelly, D.J. Larson, J. Schneir, J.P. Roberts. Spatial distribution maps for atom probe tomography, Microscopy and Microanalysis. 13 (2007) 437-447.

[6] E. Cadel, F. Vurpillot, R. Lardé, S. Duguay, B. Deconihout. Depth resolution function of the laser assisted tomographic atom probe in the investigation of semiconductors, J.Appl.Phys. 106 (2009) 044908 .

[7] G. Sha, S.P. Ringer. Effect of laser pulsing on the composition measurement of an Al-Mg-Si-Cu alloy using three-dimensional atom probe, Ultramicroscopy. 109 (2009) 580-584.

[8] Y. Zhou, C. Booth-Morrison, D.N. Seidman. On the Field Evaporation Behavior of a Model NiAl-Cr Superalloy Studied by Picosecond Pulsed-Laser Atom-Probe Tomography, Microscopy and Microanalysis. 14 (2008) 571-580.

[9] M.K. Miller, A. Cerezo, M.G. Hetherington, G.D.W. Smith, Atom probe field ion microscopy, Oxford University Press 1996.

[10] D. Hudson, G.D.W. Smith, B. Gault. Optimisation of mass ranging for atom probe microanalysis and application to the corrosion processes in $\mathrm{Zr}$-alloys, These proceedings. (2010).

[11] F. Tang, B. Gault, S.P. Ringer, J.M. Cairney. Optimization of pulsed laser atom probe (PLAP) for the analysis of nanocomposite Ti-Si-N films, Ultramicroscopy. 110 (2010) 836-843.

[12] M.K. Miller. Three-dimensional atom probes, J.Microsc. 186 (2003) 1-16.

[13] P. Ronsheim, P. Flaitz, M. Hatzistergos, C. Molella, K. Thompson, R. Alvis. Impurity measurements in silicon with D-SIMS and atom probe tomography, Appl.Surf.Sci. 255 (2008) 15471550.

[14] L. Yao, B. Gault, J.M. Cairney, S.P. Ringer. On the multiplicity of field evaporation events in atom probe: A new dimension to the analysis of mass spectra, Philosophical Magazine Letters. 90 (2010) 121-129.

[15] B. Gault, D.W. Saxey, G.D.W. Smith, M. Müller. Comparison between the field evaporation behaviour of $\mathrm{GaSb}$ and metallic materials, These proceedings. (2010).

[16] B. Gault, E.A. Marquis, D.W. Saxey, G.M. Hughes, D. Mangelinck, E.S. Toberer, et al. Highresolution nanostructural investigation of $\mathrm{Zn}_{4} \mathrm{Sb}_{3}$ alloys, Scripta Materialia. 63 (2010) 784-787. 
[17] W. Sha, L. Chang, G.D.W. Smith, L. Cheng, E.J. Mittemeijer. Some aspects of atom-probe analysis of Fe--C and Fe--N systems, Surf. Sci. 266 (1992) 416-423.

[18] T.T. Tsong, Y.S. Ng, S.V. Krishnaswamy. Quantification of atom-probe FIM data and an application to the investigation of surface segregation of alloys, Appl.Phys.Lett. 32 (1978) 778-780.

[19] O. Jagutzki, A. Cerezo, A. Czasch, R. Dorner, M. Hattas, M. Huang, et al. Multiple hit readout of a microchannel plate detector with a three-layer delay-line anode, IEEE Trans.Nucl.Sci. 49 (2002) 2477-2483.

[20] G. Da Costa, F. Vurpillot, A. Bostel, M. Bouet, B. Deconihout. Design of a delay-line positionsensitive detector with improved performance, Rev.Sci.Instrum. 76 (2005) 013304.

[21] F. De Geuser, B. Gault, A. Bostel, F. Vurpillot. Correlated field evaporation as seen by atom probe tomography, Surf. Sci. 601 (2007) 536-543.

[22] B.S. Everitt, The analysis of contingency tables, Second ed., Chapman \& Hall/CRC 1992.

[23] E. Camus, C. Abromeit. Analysis of conventional and three-dimensional atom probe data for multiphase materials, J.Appl.Phys. 75 (1994) 2373-2382.

[24] T.T. Tsong, Y. Liou. Time-of-flight energy and mass analysis of metal-helide ions and their formation and dissociation, Physical Review Letters. 55 (1985) 2180-\&.

[25] Z.M. Stepien, T.T. Tsong. Formation of metal hydride ions in low-temperature field evaporation, Surface Science. 409 (1998) 57-68.

[26] B.T. Chait, F.H. Field, Fission fragment ionization mass spectrometry: metastable decompositions. Int. J. Mass Spectrom. Ion Phys. 41 (1981) 17-29.

[27] B. Song, P.L. Cao. Geometric and electronic structures of small GaN clusters, Physics Letters A. 328 (2004) 364-374.

[28] C.M. Wei, J.M. Yuan, T.T. Tsong. Fractal dissociation dynamics of diatomic ions in a DC field, Physica A Statistical Mechanics and its Applications. 221 (1995) 297-313.

[29] R. Haydock, D.R. Kingham. Post-ionization of field-evaporated ions, Phys.Rev.Lett. 44 (1980) $1520-1523$.

[30] M. Thuvander, J.Weidow, J. Angseryd, L. Falk, F. Liu, M. Sonestedt, et al. Quantitative atom probe analysis of carbides, These proceedings. (2010).

[31] O. Nishikawa, K. Maeda, Y. Ohtani, M. Watanabe, K. Tanaka, T. Sekine, et al. Atomic level analysis of electron emitter surfaces by the scanning atom probe, Appl.Surf.Sci. 146 (1999) 398-407.

[32] F.G. Caballero, M.K. Miller, S.S. Babu, C. Garcia-Mateo. Atomic scale observations of bainite transformation in a high carbon high silicon steel, Acta Materialia. 55 (2007) 381-390.

[33] P.A. Ronsheim, M. Hatzistergos, S. Jin. Dopant measurements in semiconductors with atom probe tomography, Journal of Vacuum Science \& Technology B: Microelectronics and Nanometer Structures. 28 (2010) C1E1. 
[34] M.J. Galtrey, R.A. Oliver, M.J. Kappers, C.J. Humphreys, D.J. Stokes, P.H. Clifton, et al. Threedimensional atom probe studies of an InGaN GaN multiple quantum well structure: Assessment of possible indium clustering, Appl. Phys. Lett. 90 (2007) 061903. 


\section{Figure captions}

Figure 1: Correlation table ( $d_{i j}$ in Eq. (2.3)) for ion-pairs in the evaporation of pure aluminium. The number of ion-pairs involving each ion species is also shown. A background level of hydrogen was present in the mass spectrum at about $0.8 \%$. Diagonal entries, and those corresponding to expectation values less than five, are considered statistically insignificant and are not shown.

Figure 2: Relative abundances of ions detected together with $\mathrm{Fe}^{+}$and $\mathrm{Fe}^{2+}$ within multiple-ion events in the field evaporation of a mixed Fe-Cr oxide. The values indicate the extent to which the observed abundance of an ion deviates from the abundance that would be expected under the assumption of independent evaporation.

Figure 3: Correlation histograms for the field evaporation of gallium nitride (bin-width $0.02 \mathrm{Da}$ ). (a): Correlation between mass-charge ratios from 0 to $100 \mathrm{Da}$. (b): Magnified region of the histogram indicated by the rectangle in (a). The corresponding mass spectrum, including all detector hits, is shown below each of the correlation histograms.

Figure 4: Correlation histograms for a $\mathrm{Mg}-\mathrm{Cu}-\mathrm{Y}$ metallic glass sample under various field evaporation conditions. (a): Voltage pulsing with a nominal pulse fraction of 0.20. (b): 'Low power' laser pulsing $(0.1 \mathrm{~nJ} /$ pulse). (c): Field-evaporation induced only by a standing dc field, without pulsing. The corresponding mass spectrum, including all detector hits, is shown beneath each of the correlation histograms. The data in (c) was obtained by reducing the laser power to $0.01 \mathrm{~nJ} /$ pulse and pointing the beam well-away from the specimen. The small peaks in the mass spectrum result from a short period before the laser beam was moved, at the beginning of the analysis.

Figure 5: Molecular ion dissociation. A molecular ion of mass-charge ratio $m_{p}$ is field evaporated from a specimen surface (left) at a potential $V_{0}$. After travelling away from the surface until the potential has dropped by $V_{d}$, the ion dissociates into two or more products. One daughter ion continues to traverse the remainder of the potential drop with a mass-charge ratio of $m_{1}$. If the ion impacts on a detector at zero potential, the inferred mass-charge ratio $m_{1}^{\prime}$ will differ from the actual value according to Eq. (2.8). 
Figure 6: Dissociation tracks in a correlation histogram for field evaporation of gallium nitride. The data was obtained under 'low-power' laser pulsing $(0.046 \mathrm{~nJ} /$ pulse), in a LEAP 3000X Si instrument, which has a straight flight-path. The parametric curves (pink) superimposed on the correlation histogram are generated using Eq. (2.8), which derives from the flight-path model illustrated in Figure 5. Mass-charge ratios for the parent $\left(m_{p}\right)$ and daughter ions $\left(m_{1}\right.$ and $\left.m_{2}\right)$ are selected to provide the most appropriate fit to each histogram track, corresponding to dissociation pathways, $\mathrm{m}_{\mathrm{p}} \rightarrow \mathrm{m}_{1}+\mathrm{m}_{2}$ : (1) $\mathrm{GaN}^{2+} \rightarrow \mathrm{Ga}^{+}+\mathrm{N}^{+}$(2) $\mathrm{GaN}^{3+} \rightarrow \mathrm{Ga}^{2+}+\mathrm{N}^{+}$(3) $\mathrm{GaN}_{3}{ }^{2+} \rightarrow \mathrm{Ga}^{+}+\mathrm{N}_{3}{ }^{+}$. The grey diagonal line corresponds to the symmetry axis of the histogram, $m_{1}=m_{2}$, where the dissociation tracks terminate at the parent ion mass-charge ratio. The three subfigures (right) show details from each dissociation track. 


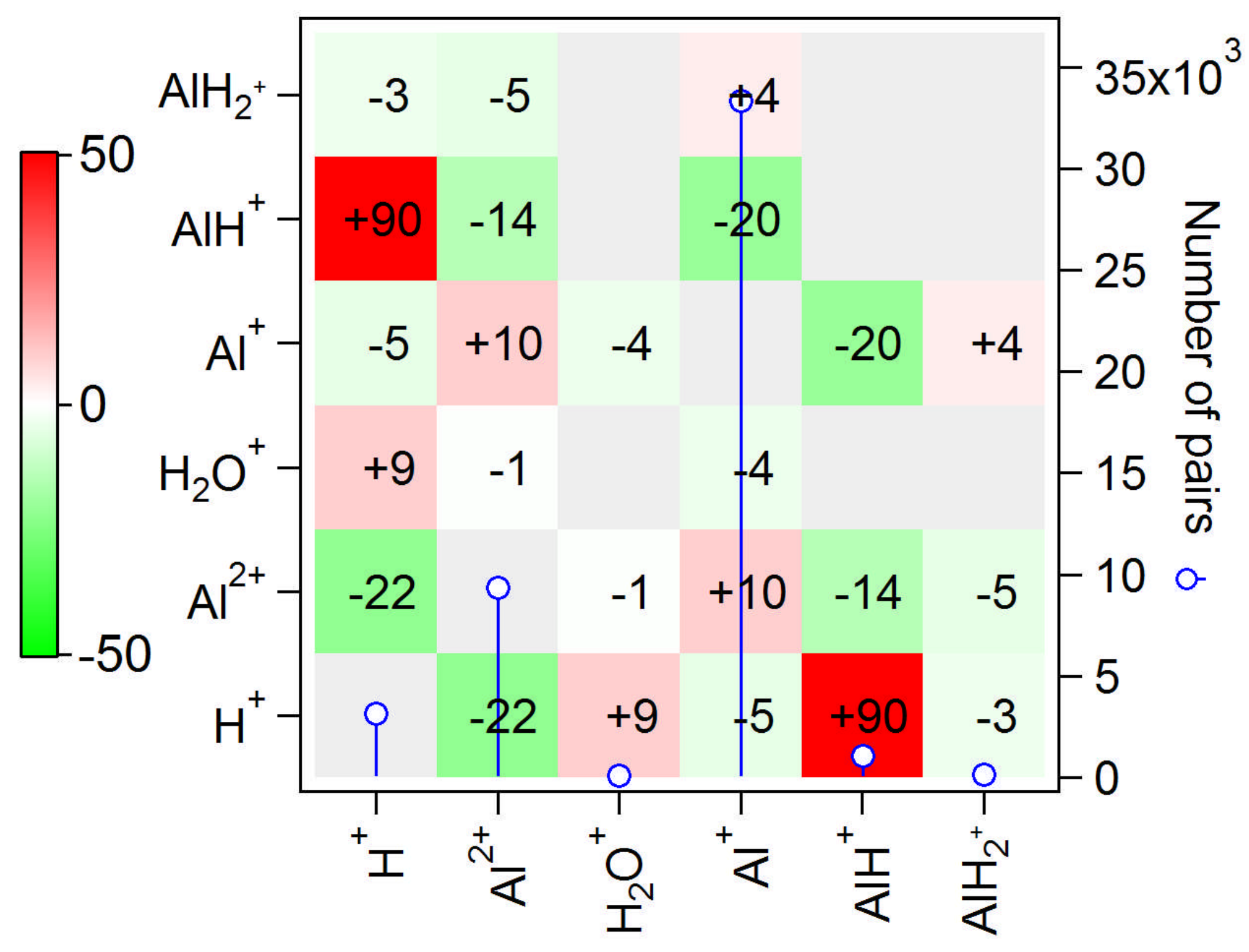

Figure 1 


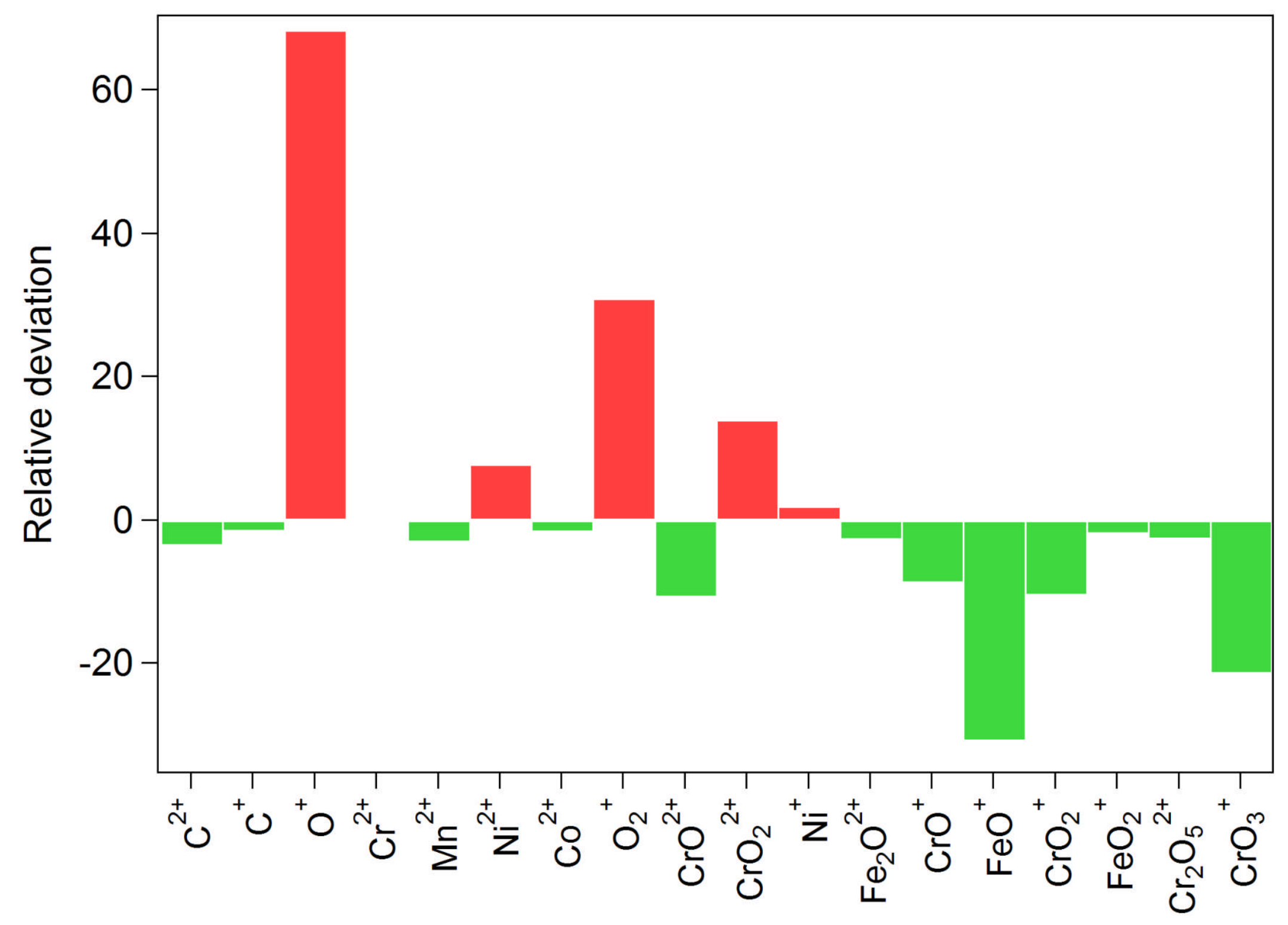

Figure 2 
(a)

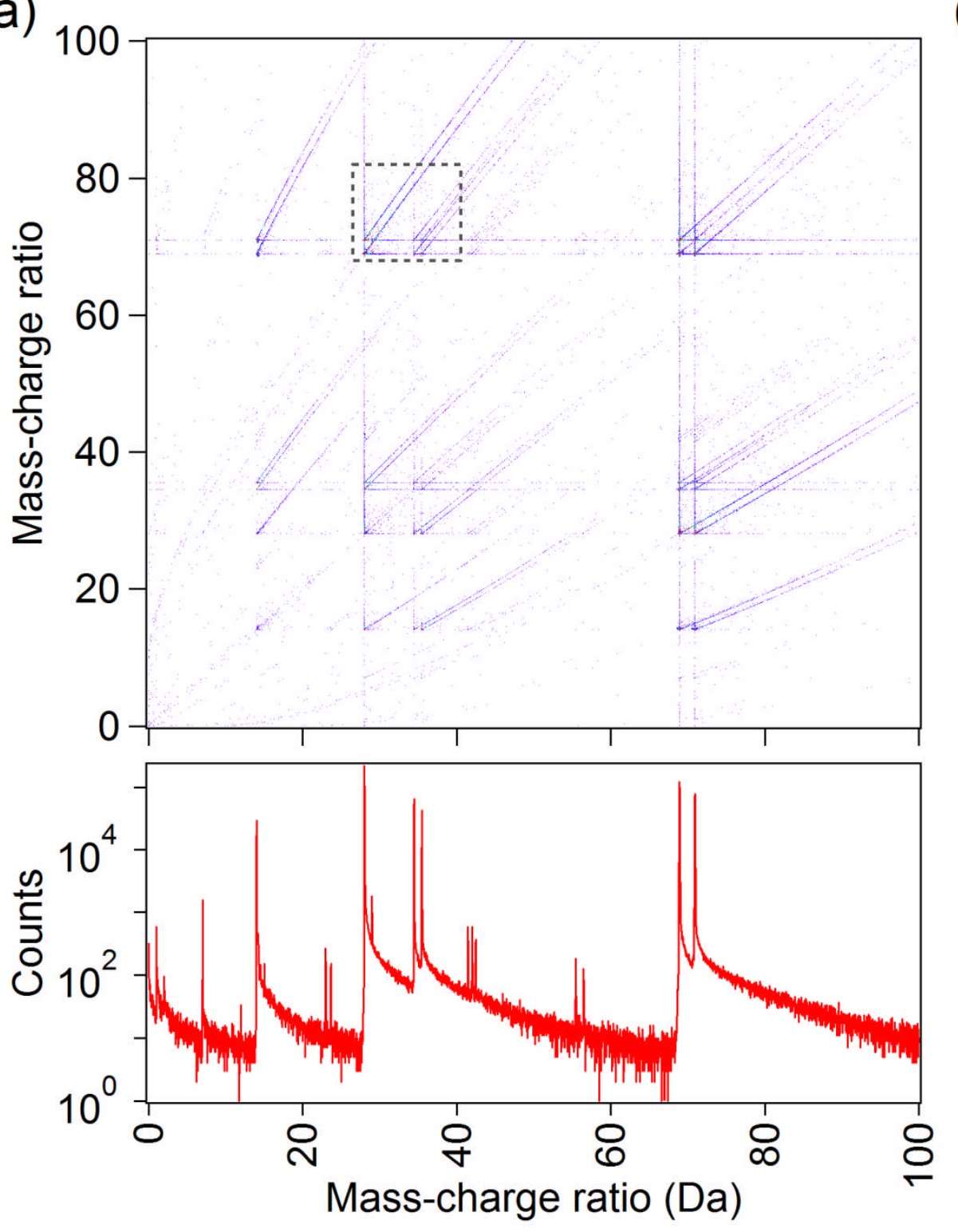

(b)
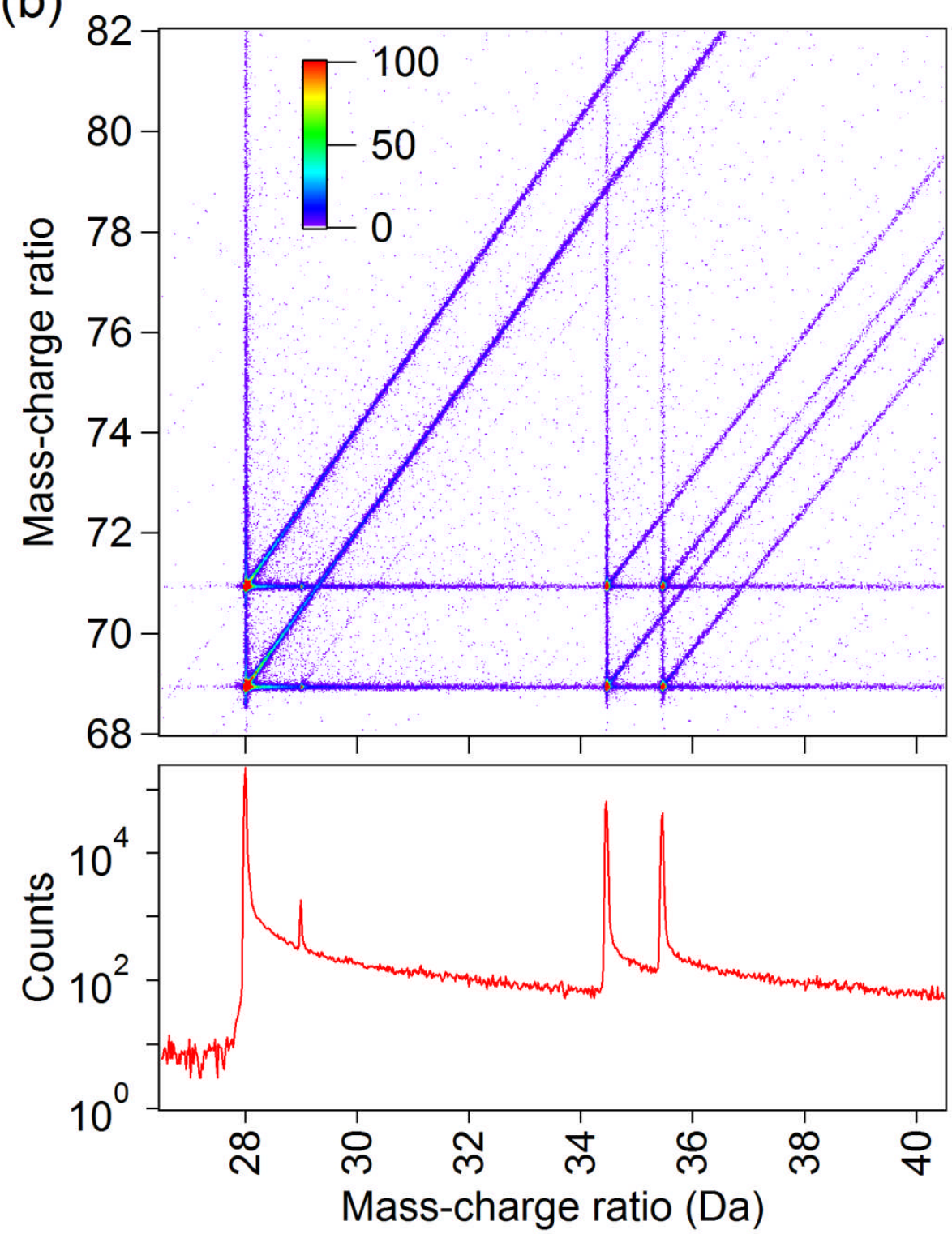

Figure 3 

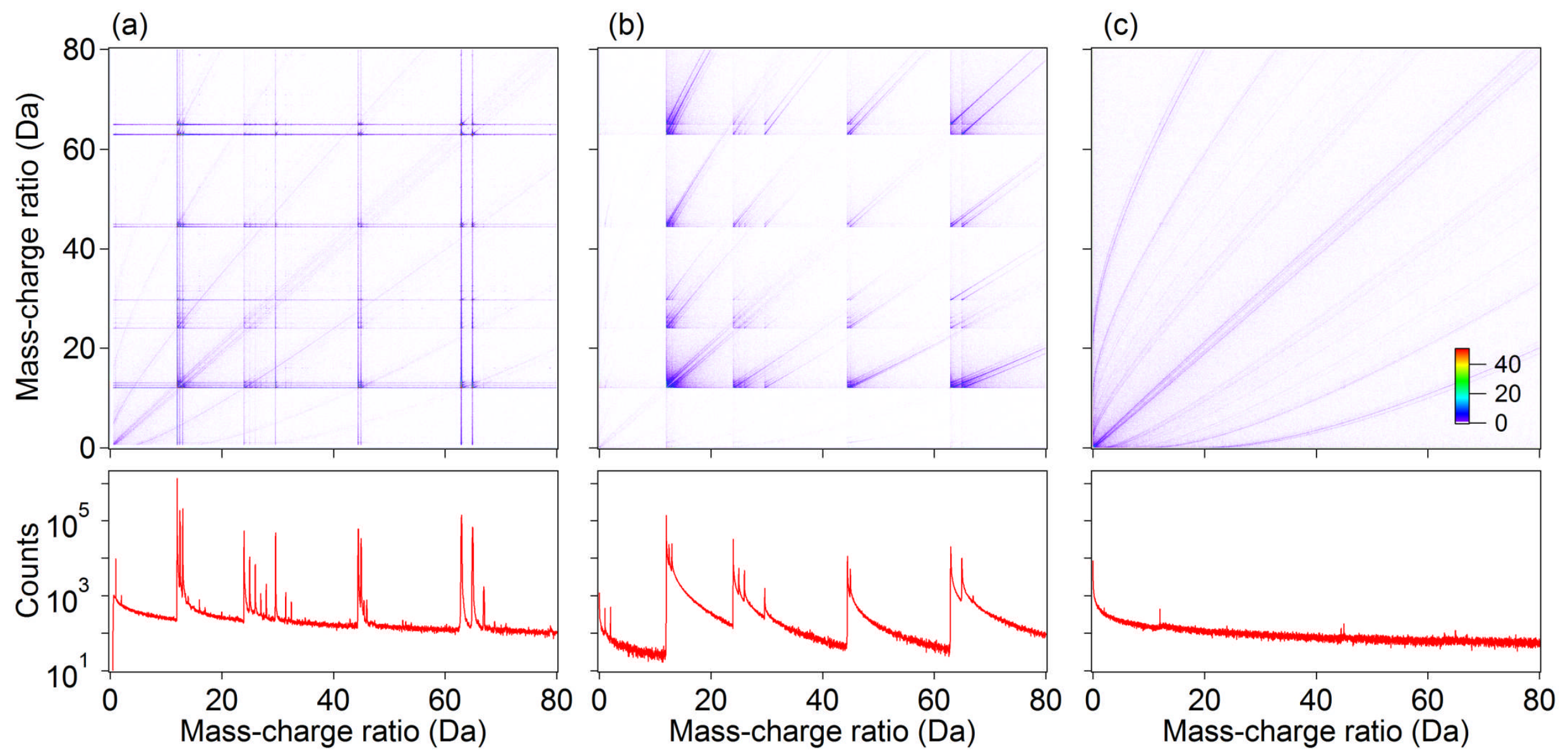

Figure 4 


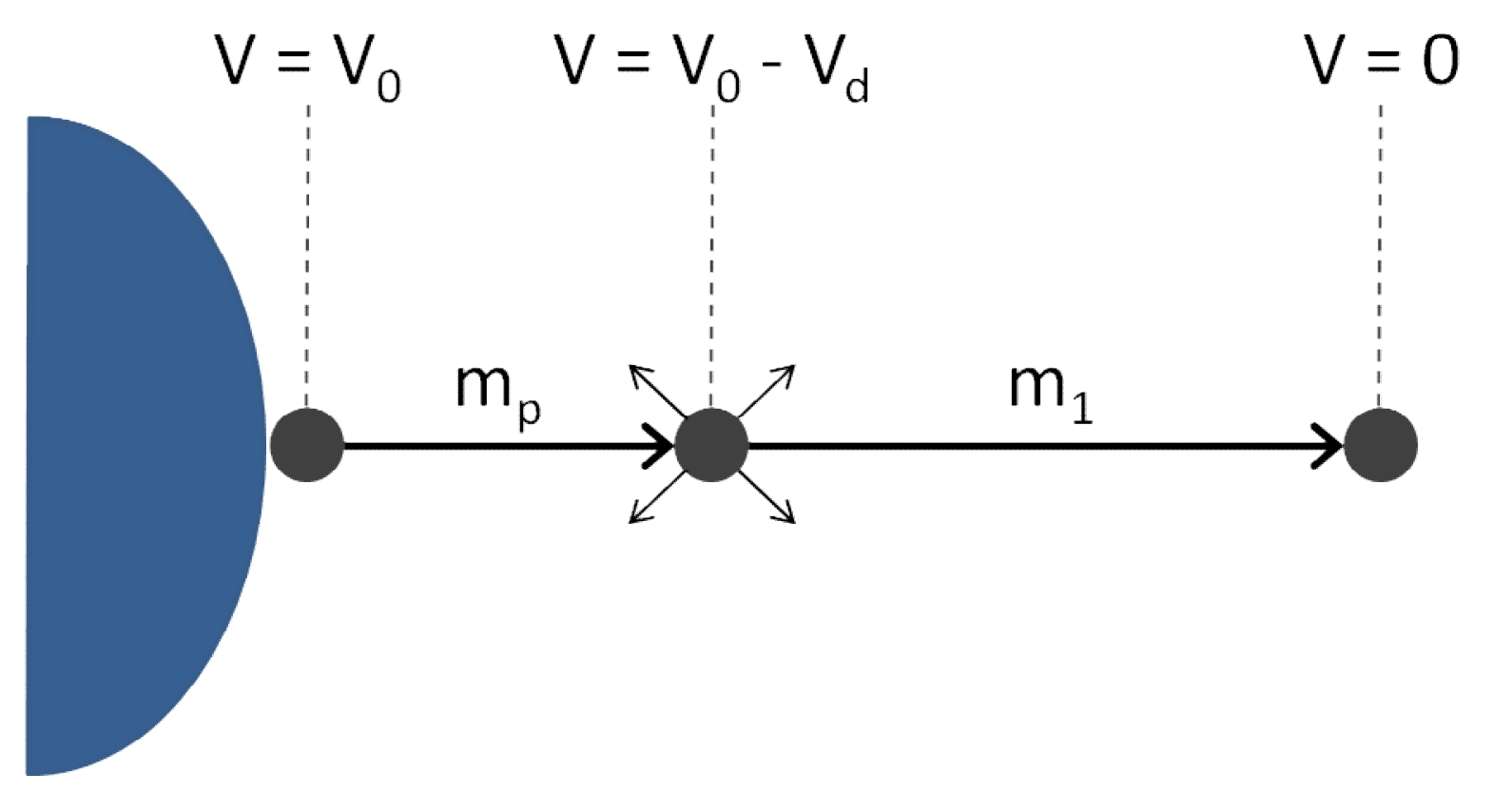

Figure 5 

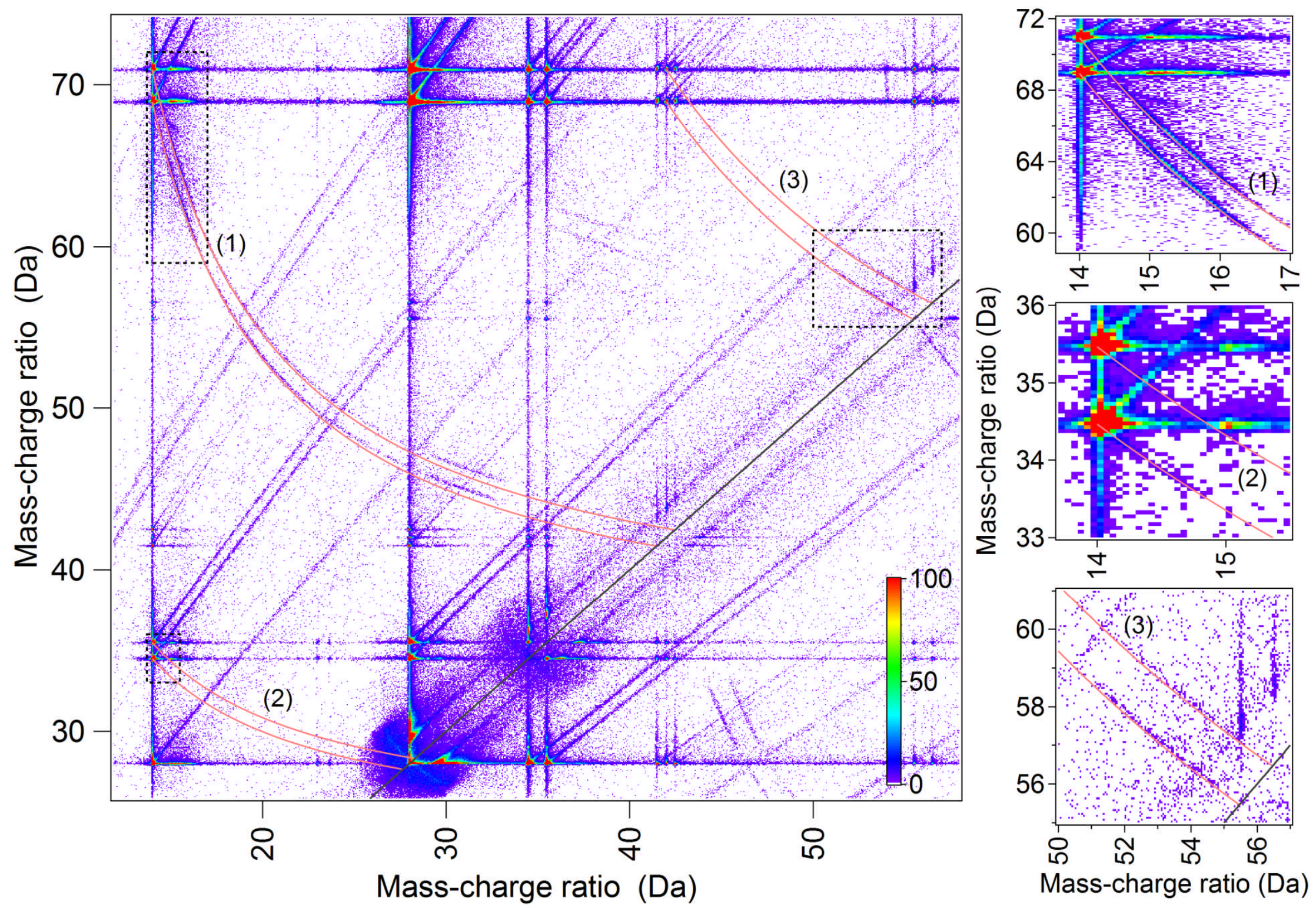

Figure 6 